\title{
ИНТЕГРАЛЬНОЕ ПРЕДСТАВЛЕНИЕ ОБЩЕГО \\ РЕШЕНИЯ И ГРАНИЧНЫЕ ЗАДАЧИ ДЛЯ \\ ОБЫКНОВЕННОГО ДИФФЕРЕНЦИАЛЬНОГО \\ УРАВНЕНИЯ СПЕЦИАЛЬНОГО ТИПА С ТРЕМЯ СЛАБО \\ СИНГУЛЯРНЫМИ ТОЧКАМИ
}

@ А.Г. Олими (А.Г. Олимов), Н.К. Охунов

Abdumanon1950@mail.ru,okhunov_73@mail.ru

УДК 517.927.21

DOI: 10.33184/mnkuomsh1t-2021-10-06.78.

В сообщении для уравнения, полученного итерированием обыкновенного дифференциального оператора первого порядка с тремя слабо сингулярными точками, найдено представление общего решения, которое применяется для исследования свойств решений, выяснения постановки и решения задач Коши - Рикье и типа линейного сопряжения.

Ключевые слова: дифференциальное уравнение специального типа, слабо сингулярная точка, общее решение, формулы обращения, свойства решений, задачи Коши - Рикье и линейного сопряжения.

Integral representation of the general solution and boundary value problems for an ordinary differential equation of a special type with three weakly singular points In the message for the equation obtained by iterating an ordinary first-order differential operator with three weakly singular points is found representation of the general solution, which is used to study the properties of solutions, formulation and solv of Cauchy - Riquier and linear conjugation problems.

Keywords: special type differential equation, weakly singular point, general solution, inversion formulas, properties of solutions, Cauchy Riquier and linear conjugation problems.

Пусть $\Gamma=(a, b),(b)=\left\{b_{1}, b_{2}, b_{3}\right\}$ - точки отрезка $\bar{\Gamma}, a=b_{1}<b_{2}<b_{3}=$ $b, \Gamma_{(b)}=\Gamma \backslash(b),(\alpha)=\left\{\alpha_{1}, \alpha_{2}, \alpha_{3}\right\}, 0<\alpha_{j}<1, j=\overline{1,3}$ - действительные числа, $n$ - натуральное число. На множестве $\Gamma_{(b)}$ рассмотрим уравнение

$$
A_{(\alpha),(b)}^{n} y=f(x) \cdot \prod_{j=1}^{3}\left|x-b_{j}\right|^{-\alpha_{j}}
$$

Олими Абдуманон Гафорзода (Олимов Абдуманон Гафорович), к.ф.-м.н., доцент, ХГУ (Худжанд, Таджикистан); Olimi Abdumanon Gaforzoda (Olimov Abdumanon Gaforovich) (Khujand State University, Khujand, Tajikistan)

Охунов Нозимджон Кобилович, ХГУ (Худжанд, Таджикистан); Okhunov Nozimjon Kobilovich (Khujand State University, Khujand, Tajikistan) 
где $p(x), q(x)$ и $f(x)$ - известные, $y(x)$ - искомая функции, а

$$
A_{(\alpha),(b)} y \equiv y^{\prime}+p(x) \prod_{j=1}^{3}\left|x-b_{j}\right|^{-\alpha_{j}} y-q(x) \prod_{j=1}^{3}\left|x-b_{j}\right|^{-\alpha_{j}}
$$

- Решение уравнения (1) определяется также как в работах [1,2].

Теорема 1. Пусть в уравнении (1) функции $p(x), q(x)$ u $f(x)$ непрерывны на отрезке $\bar{\Gamma}$ за исключением, быть может, точек $b_{j}, j=\overline{1,3}$. $B$ этих точках они могут иметь разрыв первого рода. Пусть $\Gamma=\bigcup_{i=1}^{2} \Gamma_{i}$ , $\Gamma_{i}=\left(b_{i}, b_{i+1}\right), x_{i}^{0}$ - биксированная точка промежутка $\Gamma_{i}$, далее $\Gamma_{i}^{1}=$ $=\left(b_{i}, x_{i}^{0}\right], \Gamma_{i}^{2}=\left[x_{i}^{0}, b_{i+1}\right), i=1,2$.Тогда общее решение уравнения (1), а также степени оператора $A_{(\alpha),(b)}$ от нее выражается формулой

$A_{(\alpha),(b)}^{s} y(x)=\left\{\begin{array}{ll}I_{b_{i}, s}^{\alpha_{i}+}\left[p_{i}^{1}(x), q_{i}^{1}(x), f_{i}^{1}(x), C_{i s}^{1}, \ldots, C_{i(n-1)}^{1}\right] & \text { при } x \in \Gamma_{i}^{1} \\ I_{b_{i+1}, s}^{\alpha_{i+1}-}\left[p_{i}^{2}(x), q_{i}^{2}(x), f_{i}^{2}(x), C_{i s}^{2}, \ldots, C_{i(n-1)}^{2}\right] & \text { при } x \in \Gamma_{i}^{2}\end{array}, i=1,2\right.$,

$s=\overline{0,(n-1)}$, где $p_{i}^{k}(x), q_{i}^{k}(x), f_{i}^{k}(x), k=1,2$-известные функиии а $I_{b_{i}, s}^{\alpha_{i},+}[\ldots]$ u $I_{b_{i+1}, s}^{\alpha_{i+1},-}[\ldots]$ известные интегральные операторы, соответственно определяемые по формулам (1.9) и (2.3) из работы [2, с.6,7], a $C_{i j}^{k}$, $i, k=1,2, j=\overline{0,(n-1)}$ - произвольные постоянные.

Представление (2) обладает следующими свойствами: а) группы произвольных постоянных $C_{i j}^{1}$ и $C_{i j}^{2}$, соответствующие значениям $i=1$ и $i=2, j=\overline{0,(n-1)}$ однозначно выражаются одна через другой, так что, формула зависит только от $n$ произвольных постоянных; б) представление обратимо, если известно решение уравнения (1), выражаемое формулой (2), то соответствующие ему постоянные находятся однозначHO.

Выше сказанное позволяет решить следующие задачи Коши - Рикье и типа линейного сопряжения, единственное решение которых дается при помощи формулы (2): требуется найти решение уравнения (1), подчиняющееся одному из следующих групп условий:

$$
\begin{gathered}
1)\left[A_{(\alpha),(b)}^{j} y(x)\right]_{x=b_{1}+0}=y_{1 j}^{1}, \quad\left[A_{(\alpha),(b)}^{j} y(x)\right]_{x=b_{2}+0}=y_{2 j}^{1} ; \\
2)\left[A_{(\alpha),(b)}^{j} y(x)\right]_{x=b_{2}+0}=y_{2 j}^{1}, \quad\left[A_{(\alpha),(b)}^{j} y(x)\right]_{x=b_{2}-0}=y_{1 j}^{2} ; \\
3)\left[A_{(\alpha),(b)}^{j} y(x)\right]_{x=b_{2}-0}=y_{1 j}^{2}, \quad\left[A_{(\alpha),(b)}^{j} y(x)\right]_{x=b_{3}-0}=y_{2 j}^{2} ; \\
4)\left[A_{(\alpha),(b)}^{j} y(x)\right]_{x=b_{1}+0}=y_{1 j}^{1}, \quad\left[A_{(\alpha),(b)}^{j} y(x)\right]_{x=b_{3}-0}=y_{2 j}^{2}, \quad j=\overline{0,(n-1)}
\end{gathered}
$$




$$
\begin{gathered}
5) \sum_{j=0}^{n-1} b_{i j}\left[A_{(\alpha),(b)}^{j} y(x)\right]_{x=b_{1}+0}+\sum_{j=0}^{n-1} b_{i(n+j)}\left[A_{(\alpha),(b)}^{j} y(x)\right]_{x=b_{2}-0}+ \\
+\sum_{j=0}^{n-1} b_{i(2 n+j)}\left[A_{(\alpha),(b)}^{j} y(x)\right]_{x=b_{2}+0}+\sum_{j=0}^{n-1} b_{i(3 n+j)}\left[A_{(\alpha),(b)}^{j} y(x)\right]_{x=b_{3}-0}=d_{i}, i=\overline{1,2 n},
\end{gathered}
$$

где $y_{i j}^{k}, i, k=1,2, b_{i j}$ и $d_{i}, i=\overline{1,2 n}, j=\overline{0,(n-1)}$ - известные числа.

\section{Литература}

1. Rajabov $N$. Introduction to ordinary differential equations with singular and super - singular coefficients. Dushanbe, (1998), 160p.

2. Дадоджонова М.Я.,Олимов А.Г. Интегральное представление решений и задача Коши-Рикье для двух обыкновенных дифференциальных уравнений специального типа с граничной слабо сингулярной точкой // Ученые записки. Серия естественные и экономические науки. Учредитель: Худжандский государственный университет им. Б.Г.Гафурова, 2015, №2(33), 3-10. 